\title{
Research Article \\ Optimal Geometric Mean Returns of Stocks and Their Options
}

\author{
Guoyi Zhang \\ Department of Mathematics and Statistics, University of New Mexico, Albuquerque, NM 87131, USA \\ Correspondence should be addressed to Guoyi Zhang, gzhang123@gmail.com
}

Received 23 October 2012; Accepted 9 December 2012

Academic Editor: Qing Zhang

Copyright (C 2012 Guoyi Zhang. This is an open access article distributed under the Creative Commons Attribution License, which permits unrestricted use, distribution, and reproduction in any medium, provided the original work is properly cited.

The optimal geometric mean return is an important property of an asset. As a derivative of the underlying asset, the option also has this property. In this paper, we show that the optimal geometric mean returns of a stock and its option are the same from Kelly criterion. It is proved by using binomial option pricing model and continuous stochastic models with self-financing assumption. A simulation study reveals the same result for the continuous option pricing model.

\section{Introduction}

The original question of Kelly criterion [1] is how to bet the fraction of your total wealth to maximize your long-term wealth when the odds and probabilities of a gambling game are known. Latane [2] first introduced the geometric mean investment strategy into finance and economics. As an application of generalized Kelly criterion, Latane and Tuttle [3] proposed a wealth maximizing model for building portfolios using geometric mean return. Bickel [4] discovered the relationship between optimal long run growth rate and the efficient portfolios based on the minimum variance criterion. Weide et al. [5] and Maier et al. [6] developed a strategy which maximizes the geometric mean return on portfolio investment. Similar research can be found by Ziemba [7], Elton and Gruber [8], and Bernstein and Wilkinson [9]. How to optimize the geometric mean return by the Kelly criterion becomes an important question faced by many portfolio managers and researchers.

In the literature, Kelly criterion is also known as growth optimal portfolio, capital growth theory of investment, geometric mean strategy, investment for the long run, and maximum expected log. Estrada [10] used it as geometric mean maximization (GMM) and compared the popular mean variance analysis and Kelly criterion from an empirical perspective. Merton [11] was the first one to address the dynamic portfolio choice problem 
using the idea from Kelly criterion, which becomes a well-known topic in finance. McEnally [12] provided an overview of Kelly criterion, and MacLean et al. [13] summarized desirable and undesirable properties of Kelly criterion.

Stock options are popular in many financial markets. An option is a contract between a buyer and a seller that gives the buyer right to buy or to sell a particular stock at a later day with a fixed price. A call option gives buyers right to buy stock and a put option gives buyers right to sell stock. The theoretical value of an option can be evaluated according to several models. Most of the theorems and models assume that market is free of arbitrage. Arbitrage is to make a guaranteed profit with no invested capital. Arbitrage can be considered as a sure win betting scheme such that investors can apply some certain strategy at the beginning and collect the guaranteed profit at the end. Cox et al. [14] proposed a simple discrete time binomial option pricing model for evaluating options. The celebrated Black-Scholes model [15] is a special limiting case of the binomial tree model, which assumes that there is no arbitrage opportunities and stock price is a geometric Brownian motion process.

The goal of this paper is to investigate the relationship between the optimal geometric mean returns of a stock and its option from Kelly criterion, assuming there is no betting strategy that leads to a sure win. This paper is organized as follows. In Section 2, we introduce Kelly criterion. In Section 3, we prove that the optimal geometric mean returns of a stock and its option are the same for a binomial option pricing model. Section 4 extends the study to continuous stochastic model with self-financing assumption and performs a simulation study. Section 5 gives the summary.

\section{Kelly Criterion}

Suppose the odds and the probabilities of a gambling game are known; that is, we could double our bet with probability $p_{0}$ and lose the bet with probability $q_{0}$, where $q_{0}=1-p_{0}$. The question is how to maximize our total wealth in the long run, assuming we can play the game again and again. Kelly [1] proposed to bet a fraction $l$ of the total capital each time. After $N$ bets, the total wealth $W$ is

$$
W=(1+l)^{i}(1-l)^{j} W_{0}
$$

where $W_{0}$ is the starting capital and $i$ and $j$ are the number of wins and losses among the $N$ bets, respectively. The geometric mean return over $N$ periods $G$ is $(1+l)^{i / N}(1-l)^{j / N}$. The optimal geometric mean return is $\left(2 p_{0}\right)^{i / N}\left(2-2 p_{0}\right)^{j / N}$.

More generally, let $R_{0}$ be the risk-free interest rate, and let $R_{1}$ and $R_{2}$ be two possible returns with probabilities $p_{1}$ and $p_{2}$, respectively. The corresponding excess returns are $e_{1}=$ $R_{1}-R_{0}$ and $e_{2}=R_{2}-R_{0}$. In this case, the total wealth after $N$ periods is $\left(1+R_{0}+l e_{1}\right)^{i}\left(1+R_{0}+\right.$ $\left.l e_{2}\right)^{j} W_{0}$. The optimal fraction is

$$
l_{\mathrm{opt}}=\frac{p_{1} e_{1}+p_{2} e_{2}}{-e_{1} e_{2}}\left(1+R_{0}\right)
$$

and the optimal geometric mean return is

$$
G_{\mathrm{opt}}=\left(1+R_{0}+e_{1} l_{\mathrm{opt}}\right)^{p_{1}}\left(1+R_{0}+e_{2} l_{\mathrm{opt}}\right)^{p_{2}}
$$




\section{Binomial Option Pricing Model}

In this section, we use a call option to illustrate the one-step binomial option model. We state the arbitrage free call option price in Lemma 3.1 and prove that the optimal geometric mean returns are the same for a stock and its option in Theorem 3.2, assuming the market is free of arbitrage opportunities and the one-step binomial option model is appropriate. An example is given to illustrate Theorem 3.2.

Lemma 3.1. Assume the market is free of arbitrage opportunities. The current price of a stock is $S_{1}$, and after one time period the stock either goes up to $S_{3}$ with probability $p$ or down to $S_{2}$ with probability $q=1-p$. The risk-free interest rate is $R_{0}$ and the exercise price $K$ is between $S_{2}$ and $S_{3}$. The arbitrage free call option price is

$$
C=\left(S_{1}-\frac{S_{2}}{1+R_{0}}\right) \frac{S_{3}-K}{S_{3}-S_{2}} .
$$

Lemma 3.1 states that given the no arbitrage assumption, the price of the option is unique and is not related to the probability distribution of $p$.

Theorem 3.2. Assume the market is free of arbitrage opportunities and the one-step binomial option model is appropriate. The optimal geometric mean returns are the same for a stock and its option. The optimal geometric mean returns and optimal fractions of the stock and option depend on $p$ but the ratio of optimal fractions of stock and option is not related to $p$.

Proof. By (2.2) and (2.3), the optimal fraction $\left(l_{\mathrm{opt}}^{s}\right)$ and geometric mean return $\left(G_{\mathrm{opt}}^{s}\right)$ for the stock are

$$
\begin{gathered}
l_{\mathrm{opt}}^{s}=\frac{p * e_{1}^{s}+q * e_{2}^{s}}{-e_{1}^{s} * e_{2}^{s}}\left(1+R_{0}\right), \\
G_{\mathrm{opt}}^{s}=\left(1+R_{0}+e_{1}^{s} * l_{\mathrm{opt}}^{s}\right)^{p}\left(1+R_{0}+e_{2}^{s} * l_{\mathrm{opt}}^{s}\right)^{q},
\end{gathered}
$$

where $e_{1}^{s}$ and $e_{2}^{s}$ are the corresponding excess returns for stock.

Similarly, the optimal fraction $\left(l_{\mathrm{opt}}^{o}\right)$ and geometric mean return $\left(G_{\mathrm{opt}}^{o}\right)$ for the option are

$$
\begin{gathered}
l_{\mathrm{opt}}^{o}=\frac{p * e_{1}^{o}+q * e_{2}^{o}}{-e_{1}^{o} * e_{2}^{o}}\left(1+R_{0}\right), \\
G_{\mathrm{opt}}^{o}=\left(1+R_{0}+e_{1}^{o} * l_{\mathrm{opt}}^{o}\right)^{p}\left(1+R_{0}+e_{2}^{o} * l_{\mathrm{opt}}^{o}\right)^{q},
\end{gathered}
$$

where $e_{1}^{o}$ and $e_{2}^{o}$ are the corresponding excess returns for option.

To prove the equivalence of (3.3) and (3.5), it is sufficient to show that

$$
e_{1}^{s} * l_{\mathrm{opt}}^{s}=e_{1}^{o} * l_{\mathrm{opt}}^{o}, \quad e_{2}^{s} * l_{\mathrm{opt}}^{s}=e_{2}^{o} * l_{\mathrm{opt}}^{o} .
$$

By applying (3.2) and (3.4), (3.6) reduces to $e_{1}^{s} * e_{2}^{o}=e_{1}^{o} * e_{2}^{s}$, which can be derived by Lemma 3.1. Theorem 3.2 is proved. 
Table 1: Optimal fractions and Geometric mean returns (discrete case).

\begin{tabular}{lccc}
\hline$p$ & $l_{\text {opt }}^{s}$ & $l_{\text {opt }}^{o}$ & $G_{\text {opt }}$ \\
\hline 0.70 & .4400 & .2000 & 1.1137 \\
0.72 & .5573 & .2533 & 1.1223 \\
0.74 & .6747 & .3067 & 1.1333 \\
0.76 & .7920 & .3600 & 1.1466 \\
0.78 & .9093 & .4133 & 1.1628 \\
0.80 & 1.0267 & .4667 & 1.1819 \\
\hline
\end{tabular}

Equation (3.7) gives an interesting result derived from (3.6):

$$
\frac{l_{\mathrm{opt}}^{s}}{l_{\mathrm{opt}}^{o}}=\frac{e_{1}^{o}}{e_{1}^{s}}=\frac{e_{2}^{o}}{e_{2}^{s}},
$$

that is, the ratio of optimal fractions of stock and options is not related to $p$. The following gives an example to illustrate Theorem 3.2.

Example 3.3. Suppose $S_{1}=50, S_{2}=30, S_{3}=70, R_{0}=.1$, and $K=50$. From Lemma 3.1, the price of a call option is 11.36, which is not related to $p$. Table 1 gives the optimal fractions and optimal geometric mean returns corresponding to different $p$ values using (2.2). We can see from Table 1 that the optimal fractions and geometric mean return increase as the probability $p$ increases. The ratio of the optimal fractions of stock and options is 2.2 from (3.7), which can also be derived using the data from Table 1. In order to have a higher return, we should bet a larger proportion of the capital if the probability of return $p$ is also higher.

\section{Continuous Stochastic Models}

In this section, we prove that the optimal geometric mean returns of a stock and its option are the same, when stock price is a geometric Brownian motion process using a self-financing strategy over time. A small simulation study is conducted to study the optimal geometric mean returns for a geometric Brownian motion process.

\subsection{Continuous Stochastic Models with Self-Financing Assumption}

Let stock price $P=\left(P_{t}\right)_{t \geq 0}$ follow a geometric Brownian motion. Let the price of a call option $O=\left(O_{t}\right)_{t \geq 0}$ be given by Black-Scholes formula, hence no arbitrage opportunity with maturity $T>0$ and strike price $K>0$. Consider a financial market containing a risk-free money market account whose value is given by $B=\left(B_{t}\right)_{t \geq 0}$. For all $t \geq 0$, the values of these processes can be modeled as solutions of the following stochastic differential equations $d P_{t}=u P_{t} d t+$ $\sigma P_{t} d \beta_{t}, d O_{t}=b_{t} O_{t} d t+a_{t} O_{t} d \beta_{t}$, and $d B_{t}=r B_{t} d t$, where $\beta=\left(\beta_{t}\right)_{t \geq 0}$ is a standard Brownian motion and the drift rate $u$ and the volatility $\sigma$ are both positive constants. It follows that for all $t \in[0, T], O_{t}=C\left(t, P_{t}\right)$, where $C(t, p)=p \Phi\left(d_{1}(t, p)\right)-k e^{-r(T-t)} \Phi\left(d_{2}(t, p)\right)$ is the Black and 
Scholes [7] formula for the price of a call option with $d_{1}(t, p)=\left(\ln (p / k)+\left(r+(1 / 2) \sigma^{2}\right)(T-\right.$ $t)) / \sigma \sqrt{T-t}$, and $d_{2}(t, p)=d_{1}-\sigma \times \sqrt{T-t}$. An application of Itô's formula yields

$$
\begin{aligned}
d O_{t} & =\frac{\partial C}{\partial t}\left(t, P_{t}\right) d_{t}+\frac{\partial C}{\partial p}\left(t, P_{t}\right) d P_{t}+\frac{1}{2} \frac{\partial^{2} C}{\partial p^{2}}\left(t, P_{t}\right) d\langle x\rangle_{t} \\
& =\left(\frac{\partial C}{\partial t}\left(t, P_{t}\right)+u P_{t} \frac{\partial C}{\partial p}\left(t, P_{t}\right)+\frac{1}{2} \sigma^{2} P_{t}^{2} \frac{\partial^{2} C}{\partial p^{2}}\left(t, P_{t}\right)\right) d t+\sigma P_{t} \frac{\partial C}{\partial p}\left(t, P_{t}\right) d \beta_{t}
\end{aligned}
$$

for all $t \geq 0$.

Now we have

$$
\begin{aligned}
b_{t} & =\frac{(\partial C / \partial t)\left(t, P_{t}\right)+u P_{t}(\partial C / \partial p)\left(t, P_{t}\right)+(1 / 2) \sigma^{2} P_{t}^{2}\left(\partial^{2} C / \partial p^{2}\right)\left(t, P_{t}\right)}{C\left(t, P_{t}\right)} \\
& =\frac{(u-r) P_{t}(\partial C / \partial p)\left(t, P_{t}\right)+r C\left(t, P_{t}\right)}{C\left(t, P_{t}\right)}
\end{aligned}
$$

and $a_{t}=\sigma P_{t}(\partial C / \partial p)\left(t, P_{t}\right) / C\left(t, P_{t}\right)$. Hence

$$
\frac{b_{t}-r}{a_{t}}=\frac{(u-r) P_{t}(\partial C / \partial p)\left(t, P_{t}\right)+r C\left(t, P_{t}\right)-r}{\sigma P_{t}(\partial C / \partial t)\left(t, P_{t}\right) / C\left(t, P_{t}\right)}=\frac{u-r}{\sigma}
$$

Let $l^{s}=\left(l_{t}^{s}\right)_{t \geq 0}$ denote the fraction invested in a stock using self-financing strategy over time. For all $t \geq 0$, the wealth $w^{l^{s}}=\left(w_{t}^{l^{s}}\right)_{t \geq 0}$ can be expressed as

$$
w_{t}^{l^{s}}=\frac{l_{t}^{S} w_{t}^{l^{s}}}{P_{t}} P_{t}+\frac{\left(1-l_{t}^{S}\right) w_{t}^{l^{s}}}{B_{t}} B_{t}
$$

The self-financing property of the portfolio then yields

$$
d w_{t}^{l^{s}}=\frac{l_{t} w_{t}^{l^{s}}}{P_{t}} d P_{t}+\frac{\left(1-l_{t}\right) w_{t}^{l^{s}}}{B_{t}} d B_{t}=\left(r+(u-r) l_{t}^{S}\right) w_{t}^{l^{s}} d t+\sigma l_{t}^{S} w_{t}^{l^{s}} d \beta_{t}
$$

Next, we use Itô's formula to obtain

$$
\begin{aligned}
d \ln w_{t}^{l^{s}} & =\frac{1}{w_{t}^{l^{s}}} d w_{t}^{l^{s}}-\frac{1}{2} \frac{1}{\left(w_{t}^{l^{s}}\right)^{2}} d\left\langle w^{l^{s}}\right\rangle_{t} \\
& =\left(r+(u-r) l_{t}^{s}-\frac{1}{2} \sigma^{2}\left(l_{t}^{s}\right)^{2}\right) d t+\sigma l_{t}^{s} d \beta_{t}
\end{aligned}
$$

for all $t \geq 0$. 
Now, the optimal geometric mean return can be obtained by the trading strategy $\tilde{l}^{s}=\left(\tilde{l}_{t}^{s}\right)_{t>0}$ that maximizes the growth rate $g_{t}\left(l_{t}^{s}\right)$ that is equivalent to $g_{t}\left(l_{t}^{s}\right)=r+(u-r) l_{t}^{s}-$ $(1 / 2) \sigma^{2}\left(l_{t}^{S}\right)^{2}$, for all $t \geq 0$. Set $g_{t}^{\prime}\left(l_{t}^{S}\right)=u-r-\sigma^{2} l_{t}^{s}=0$ to get the optimal fraction $\widetilde{l}^{s}=(u-r) / \sigma^{2}$. Putting this expression into (4.5), we obtain the following stochastic differential equation for the optimal wealth:

$$
d w_{t}^{\tilde{l}^{s}}=\left(r+\left(\frac{u-r}{\sigma}\right)^{2}\right) w_{t}^{\tilde{l}^{s}} d t+\frac{u-r}{\sigma} w_{t}^{l^{s}} d \beta_{t}
$$

Let $l^{0}=\left(l_{t}^{0}\right)_{t \geq 0}$ denote the fraction invested in a call option of the stock and use selffinancing strategy over time. The wealth $w^{l^{0}}=\left(w_{t}^{l^{0}}\right)_{t \geq 0}$ can be expressed as

$$
w_{t}^{l^{0}}=\frac{l_{t}^{0} w_{t}^{l^{0}}}{O_{t}} O_{t}+\frac{\left(1-l_{t}^{0}\right) w_{t}^{l^{0}}}{\beta_{t}} \beta_{t}
$$

for all $t \geq 0$. Repeat the previous process to discover that the optimal fraction $\widetilde{l}^{0}=\left(\widetilde{l}_{t}^{0}\right)_{t>0}=$ $\left(b_{t}-r\right) / a_{t}^{2}$ for all $t \geq 0$, and the stochastic differential equation for the optimal wealth is as follows:

$$
\begin{aligned}
d w_{t}^{\tilde{I}^{0}} & =\left(r+\left(\frac{b_{t}-r}{a_{t}}\right)^{2}\right) w_{t}^{\tilde{I}^{0}} d t+\frac{b_{t}-r}{a_{t}} w_{t}^{{\tilde{\tau^{0}}}^{0}} d \beta_{t} \\
& =\left(r+\left(\frac{u-r}{\sigma}\right)^{2}\right) w_{t}^{\tilde{l}^{0}} d t+\left(\frac{u-r}{\sigma}\right) w_{t}^{\tilde{0}^{0}} d \beta_{t}
\end{aligned}
$$

for all $t \geq 0$ by virtue of (4.3). Hence, the optimal geometric mean returns of a stock and its option are exactly the same.

\subsection{Simulation Studies}

A popular model used for option pricing is as follows:

$$
P(t)=P_{0} * e^{Y(t)},
$$

where $P(t)$ is the price of a stock at time $t, P_{0}$ is the initial price of the stock, and $Y(t)>0$ is a Brownian motion process with drift coefficient $\mu$ and variance parameter $\sigma^{2} . P(t)$ is called a geometric Brownian motion process. In this case, the price of the call option can be calculated by Black-Scholes formula as follows:

$$
C=P_{0} * \Phi\left(d_{1}\right)-K * e^{-R_{0} * t} * \Phi\left(d_{2}\right),
$$

where $\Phi$ is the standard normal distribution function, $d_{1}=\left(\log \left(P_{0} / K\right)+\left(R_{0}+\sigma^{2} / 2\right) * t\right) / \sigma * \sqrt{t}$, and $d_{2}=d_{1}-\sigma * \sqrt{t}$. The option price is related to variance parameter $\sigma$ but not related to 
Table 2: Optimal fractions and Geometric mean returns (continuous case).

\begin{tabular}{llccccc}
\hline$\mu$ & $l_{\mathrm{opt}}^{s}$ & $l_{\mathrm{opt}}^{o}$ & $\log \left(G_{\mathrm{opt}}^{s}\right)$ & $\log \left(G_{\mathrm{opt}}^{o}\right)$ & $G_{\mathrm{opt}}^{s}$ & $G_{\mathrm{opt}}^{o}$ \\
\hline 0.05 & .21 & .05 & 0.099 & .098 & 1.104 & 1.103 \\
0.1 & .53 & .15 & .117 & .113 & 1.124 & 1.120 \\
0.15 & .85 & .25 & .151 & .143 & 1.163 & 1.154 \\
\hline
\end{tabular}

drift coefficient $\mu$. However, $\mu$ is important in calculating the optimal fractions and geometric mean returns.

Suppose the excess return $e$ has a continuous probability distribution $f$. The generalized Kelly criterion is to bet an optimal fraction of the total capital such that geometric mean return reaches its maximum. This is equivalent to select $l$ by maximizing $\log (G)$, with $\log (G)=E\left(\log \left(1+R_{0}+l * e\right)\right)$. There is no close form for optimal fractions and geometric mean returns in this case but we can use Monte Carlo simulations to estimate $l_{\text {opt }}$ and $G_{\text {opt }}$. The following are listed steps for simulation study:

(1) Calculate price of option C using Black-Scholes formula (4.11).

(2) Generate $r$ independent samples $\Upsilon(t)$ from normal distribution with mean $\mu * t$ and variance $\sigma^{2} * t$.

(3) Calculate stock price $P(t)$ at time $t$ using (4.10).

(4) Calculate excess returns for the stock $e^{s}=P(t) / P_{0}-1-R_{0}$.

(5) Calculate excess returns for the option $e^{o}=[P(t)-K]_{+} / C-1-R_{0}$ where $[x]_{+}=x$ if $x>0$ and $[x]=0$ if $x<0$.

(6) Setting a grid of $l$, for each $l$, the corresponding $\log (G)=1 / r * \sum_{j=1}^{r} \log \left(1+R_{0}+l * e_{j}\right)$. Find the maximum $\log (G)$. The corresponding $l$ is the optimal fraction $l_{\text {opt }}$ and the optimal geometric mean return is $e^{\log (G)}$.

Table 2 gives a simulation study under $\sigma=.4, t=1, P_{0}=100, R=.1$, and $K=90$. We can see that the optimal fractions and geometric mean return increase as $\mu$ increases. The optimal geometric mean returns of a stock and its option are approximately the same.

\section{Concluding Remarks}

In this research, we show that the optimal geometric mean returns of a stock and its option are the same from Kelly criterion. It is proved by using binomial option pricing model and continuous stochastic models with self-financing assumption. It is shown to be approximately true for the continuous option pricing model by simulation studies. For the discrete case, we also show that the ratio of the optimal fractions of a stock and its option is not related to the probability distribution of the return. This means that we can use a small amount of options to replace the underlying asset without changing the optimal geometric mean return and knowing the probability distribution of the return. Hence, in practice, either there are sure win chances or the prices of options are more expensive than their theoretical values. Otherwise, one should always hold more uncorrelated options instead of stocks. 


\section{Acknowledgment}

The author wants to thank the referees for their careful reading of the paper and helpful comments.

\section{References}

[1] J. L. Kelly Jr, "A new interpretation of information rate," The Bell System Technical Journal, vol. 35, pp. 917-926, 1956.

[2] H. Latane, "Criteria for choice among risky ventures," Journal of Political Economy, vol. 67, no. 2, pp. 144-155, 1959.

[3] H. Latane and D. Tuttle, "Criteria for portfolio building," Journal of Finance, vol. 22, no. 3, pp. 359-373, 1967.

[4] S. Bickel, "Minimum variance and optimal asymptotic portfolios," Management Science, vol. 16, no. 3, pp. 221-226, 1969.

[5] J. H. V. Weide, D. W. Peterson, and S. F. Maier, "A strategy which maximizes the geometric mean return on portfolio investments," Management Science, vol. 23, no. 10, pp. 1117-1123, 1977.

[6] S. F. Maier, D. W. Peterson, and J. H. V. Weide, "A monte carlo investigation of characteristics of optimal geometric mean portfolios," The Journal of Financial and Quantitative Analysis, vol. 12, no. 2, pp. 215-233, 1977.

[7] W. Ziemba, "Note on optimal growth portfolios when yields are serially correlated," Journal of Financial and Quantitative Analysis, vol. 7, no. 4, pp. 1995-2000, 1972.

[8] E. Elton and M. Gruber, "On the maximization of the geometric mean with lognormal return distribution," Management Science, vol. 21, no. 4, pp. 483-488, 1974.

[9] W. Bernstein and D. Wilkinson, "Diversification, rebalancing, and the geometric mean frontier," Social Science Research Network, Working Paper Series, 1997, http:/ /ssrn.com/abstract=53503.

[10] J. Estrada, "Geometric mean maximization: an overlooked portfolio approach?" Journal of Investing, vol. 19, no. 4, pp. 134-147, 20102010.

[11] R. C. Merton, "Optimum consumption and portfolio rules in a continuous-time model," Journal of Economic Theory, vol. 3, no. 4, pp. 373-413, 1971.

[12] R. McEnally, "Latanes bequest: the best of portfolio strategies," Journal of Portfolio Management, vol. 12, pp. 21-30, 1986.

[13] L. MacLean, W. Ziemba, and G. Blazenko, "Growth versus security in dynamic investment analysis," Management Science, vol. 38, no. 11, pp. 1562-1585, 1992.

[14] J. C. Cox, S. A. Ross, and M. Rubinstein, "Option pricing: a simplified approach," Journal of Financial Economics, vol. 7, no. 3, pp. 229-263, 1979.

[15] F. Black and M. Scholes, "The pricing of options and corporate liabilities," Journal of Political Economy, vol. 81, no. 3, pp. 637-654, 1973. 


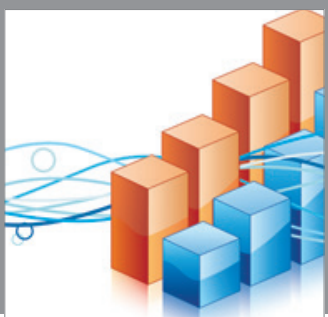

Advances in

Operations Research

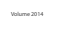

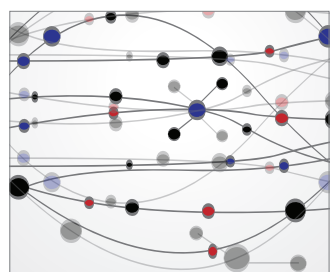

\section{The Scientific} World Journal
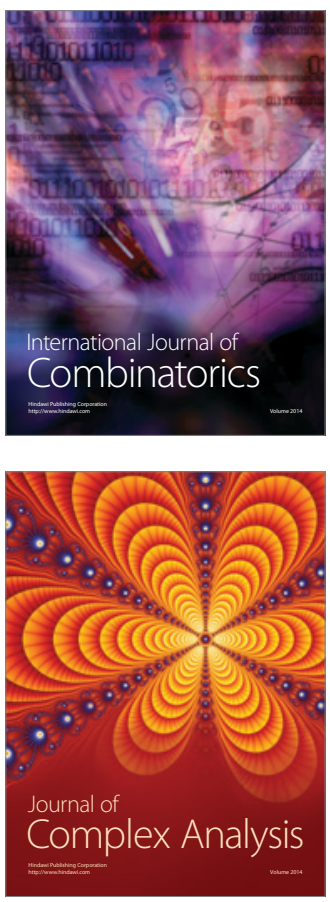

International Journal of

Mathematics and

Mathematical

Sciences
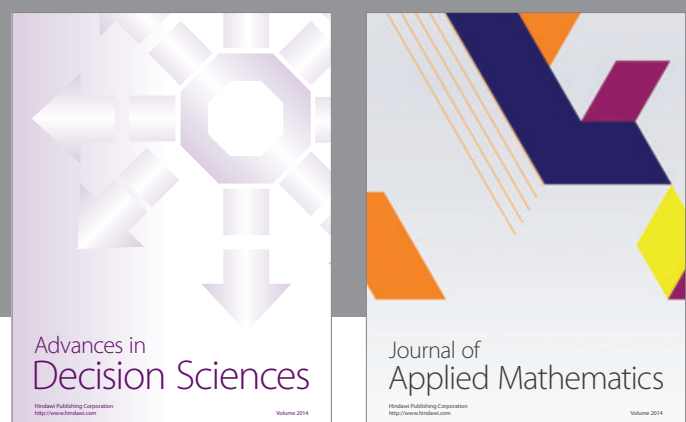

Journal of

Applied Mathematics
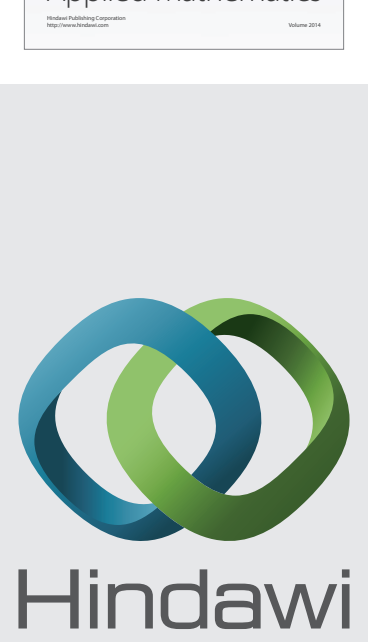

Submit your manuscripts at http://www.hindawi.com
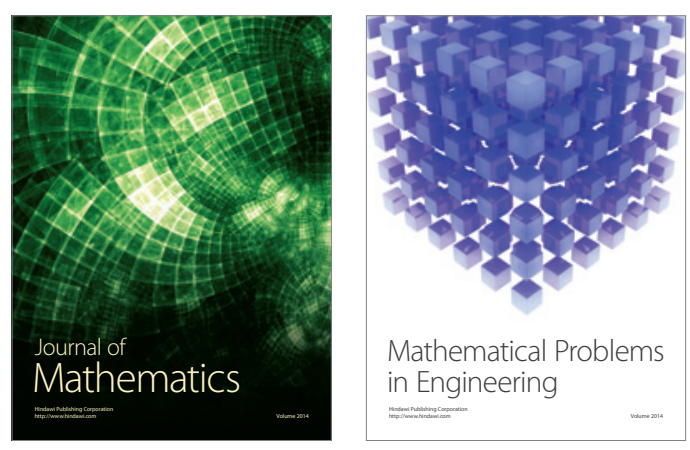

Mathematical Problems in Engineering
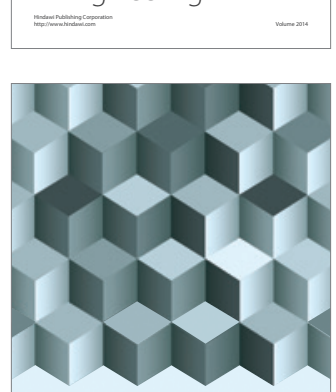

Journal of

Function Spaces
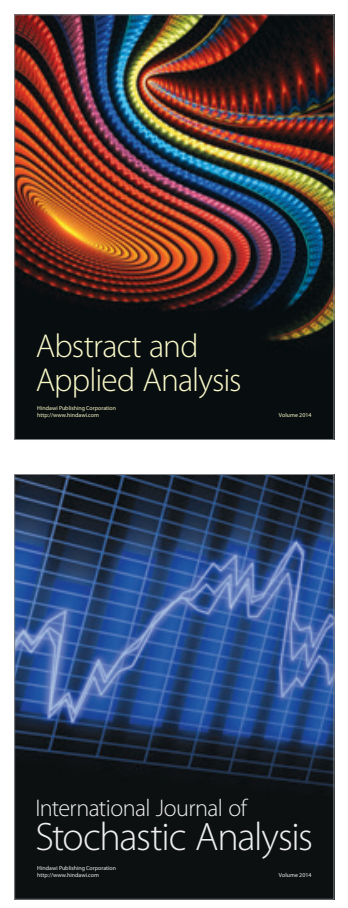

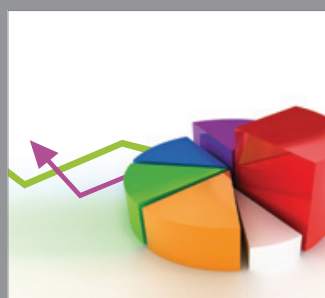

ournal of

Probability and Statistics

Promensencen
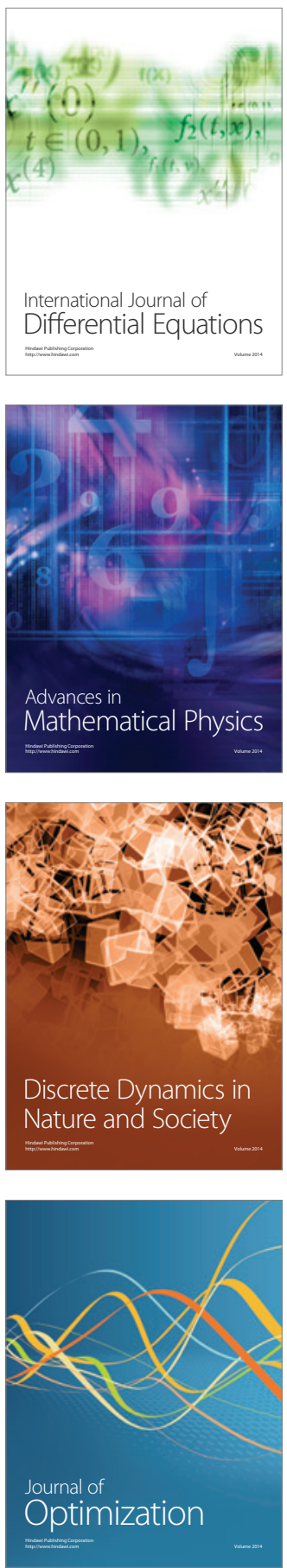